\title{
Scleral Tonometry Precision During Scleral Lens Wear: A Pilot Study
}

\author{
Allen Y Ganjei (D) \\ Gabriel GL Shlager ${ }^{2}$ \\ Daniel Brocks (iD ${ }^{3}$ \\ 'Department of Medical Education, \\ Drexel University College of Medicine, \\ Philadelphia, PA, USA; ${ }^{2}$ Department of \\ Medical Education, Tufts University \\ School of Medicine, Boston, MA, USA; \\ ${ }^{3}$ Department of Ophthalmology, \\ BostonSight, Needham, MA, USA
}

Purpose: To evaluate the reproducibility, and therefore the utility, of using traditional tonometry devices for measuring intraocular pressure (IOP), while a prosthetic replacement of the ocular surface ecosystem device (PD) or scleral lens is applied to the eye.

Patients and Methods: Twenty subjects (40 eyes) with keratoconus were enrolled. With PD applied, the first 10 consecutive patients had IOP measured multiple times with a handheld tonometer (Tono-Pen AVIA, Reichert, Depew, NY) on the superotemporal sclera $1 \mathrm{~mm}$ posterior to the PD edge. This identical procedure was repeated for the next 10 consecutive patients with a pneumatonometer (Model 30, Reichert, Depew, NY). Once three reliable measurements, as defined by the study protocol, were obtained for an eye, the procedure was repeated with the same tonometer device on the fellow eye.

Results: The mean standard deviation for reliable IOP measurements was $\pm 2.92 \mathrm{mmHg}$, median (IQR) of 2.62 (1.68 to 3.53$) \mathrm{mmHg}$ in the handheld tonometer group and \pm 1.98 $\mathrm{mmHg}$ in the pneumatonometer group. There was no statistically significant difference between the groups $(p=0.07)$. The mean IOP range for the reliable IOP measurements was $5.5 \pm 3.80 \mathrm{mmHg}$, median (IQR) of 5 (3 to 7) $\mathrm{mmHg}$ for the handheld tonometer group and $3.71 \pm 1.12 \mathrm{mmHg}$ in the pneumatonometer group. There was no statistically significant difference between the groups $(\mathrm{p}=0.06)$.

Conclusion: Handheld tonometry and pneumatonometry have poor reproducibility when used to measure scleral IOP in keratoconus patients, while a PD is applied to the eye. An alternative research model and methodology should be investigated and confirmed to have precision prior to proceeding with further analysis of any relationship between scleral lens wear and IOP.

Keywords: intraocular pressure, sclera, tonometer, reproducibility, reliability

\section{Introduction}

In recent years, clinicians have sought to determine whether the application and wear of a prosthetic replacement of the ocular surface ecosystem (PROSE, BostonSight, Needham MA) or scleral lens induces any change in intraocular pressure (IOP). ${ }^{1-6}$ Prosthetic replacement of the ocular surface ecosystem (PD) is an integrated treatment that includes designing, manufacturing and fitting a customized medical ocular surface prosthetic device (PD), comprehensive application and removal training, and ongoing collaborative optimization of optometric and ophthalmic care. The PD is manufactured using a gas-permeable plastic material (typically fluorosilicone acrylate) which is customized to each eye using a web-based design software (FitConnect, BostonSight, Needham MA) and mechanical lathe. ${ }^{7}$ The PD medical indications include: promoting ocular surface
Correspondence: Daniel Brocks

Tel + I 78I 7267337

Fax +17817267310

Email dbrocks@bostonsight.org 
comfort, supporting ocular surface health and achieving improved visual acuity. When applied to the eye, the device rests on the conjunctiva overlying the sclera, vaults over the cornea and is traditionally filled with preservative-free normal saline. This fluid reservoir remains between the posterior surface of the device and the anterior surface of the cornea throughout the daily wear time. Comparatively speaking, traditional scleral lenses function in a similar manner to the PD; however, commercial scleral lens customization options are more limited.

One glaring obstacle in prior and future studies attempting to address the question of any induced IOP changes with scleral lens wear is how a clinician can properly, accurately, and reproducibly measure IOP, while a PD or scleral lens is on the eye. The traditional standard accepted approach of corneal applanation tonometry requires that a scleral lens be removed. No particular tonometry device on the market is currently specifically designed or labelled for measuring IOP on the sclera, while a lens is applied to the eye. Although prior clinical trials have utilized scleral tonometry to investigate IOP changes in scleral lens wear, to our knowledge, no prior study has focused an investigation on the basic foundational question of the precision (reproducibility) of traditional tonometry instruments when used on the sclera with a PD or scleral lenses applied. ${ }^{1}$

Here, we report data and analysis for the first two instruments in our ongoing research to investigate the precision of various types of tonometers when utilized in clinical trials to measure scleral tonometry, while a PD or scleral lens is applied to the eye. This pilot study has the singular focus of investigating whether this commonly used procedure to measure IOP during scleral lens wear has adequate reproducibility by evaluating consecutive scleral tonometry measurements on each eye, while a lens is applied and analyzing particularly the range and standard deviation.

\section{Materials and Methods Study Design}

This prospective pilot clinical study investigated the precision of measuring intraocular pressure, while a PD is on the eye. Twenty consecutive subjects were enrolled between $8 / 5 / 2020$ and $3 / 16 / 2021$ and evaluated by the same practitioner at one center. Participants were 18 years of age or older and were recruited from a pool of current clinic patients who carried the diagnosis of corneal ectasia and were established bilateral wearers of a PD with diameters of $18.0 \mathrm{~mm}$ or greater and an optimized fit (as determined by their current dispensing optometrist and confirmed at the study slit-lamp examination). Pertinent exclusion criteria for potential participants included pregnancy or nursing, confounding health conditions, current employment by the clinic, ocular surgery in the last 12 weeks, suspected or confirmed glaucoma, ocular hypertension, or a history of prior glaucoma surgery.

Following consent and enrollment, each subject participated in the single study visit the same day. At the study visit, medical and ophthalmic histories were collected, followed by visual acuity evaluation, PD fit assessment, and slit-lamp examination. Following removal of the PD, corneal IOP was measured with a handheld tonometer (Tono-Pen AVIA, Reichert, Depew, NY) using the standard technique of central corneal applanation. This corneal IOP measurement served only as a safety parameter to ensure a normal eye pressure was present prior to proceeding further with the study visit. This data was not collected for analysis of device precision. To proceed with the study visit, both eyes were required to have a normal corneal IOP ( $\leq 21 \mathrm{mmHg}$ ). The PD was re-applied to both eyes. Measurement of intraocular pressure with the scleral lens applied was then immediately initiated as described below.

The first consecutive 10 subjects were placed in cohort one. In cohort one, the handheld tonometer was used to measure the IOP on the superotemporal sclera approximately $1 \mathrm{~mm}$ posterior to the edge of the PD. A maximum of 5 investigator attempts were allowable to try to obtain 3 reliable measurements, defined as having a confidence interval of $90 \%$ or higher on the instrument read-out. Five to ten seconds were taken between each measurement. One measurement was defined as a complete result or attempting to measure until the machine was timed out. Once 3 measurements were achieved with the specific confidence interval goal, the scleral tonometry intake on that particular eye was completed and the practitioner proceeded with the same procedure on the fellow eye.

The second consecutive 10 subjects were placed in cohort two. In cohort two, the pneumatonometer (Model 30, Reichert, Depew, NY) was used to measure IOP on the superotemporal sclera approximately $1 \mathrm{~mm}$ posterior to the edge of the PD. Maximum of ten investigator attempts were allowable to try to obtain 3 reliable measurements, defined as having a standard deviation of $2 \mathrm{mmHg}$ or lower on the instrument read-out. Five to ten seconds were taken between each measurement. A measurement 
was defined as a complete result or attempting to measure until the machine was timed out. Once 3 measurements were achieved with the specific standard deviation goal, the scleral tonometry intake on that particular eye was completed and the practitioner proceeded with the same procedure on the fellow eye.

Of note, correct calibration of each instrument was ensured prior to the study per individual manufacturer's standards. The eyes were re-examined at the conclusion of every study visit to ensure no changes had occurred to the ocular surface during the study visit.

\section{Tonometers and Technique}

The devices and techniques utilized to obtain intraocular pressure are described below. Calibration and methodology used were based on manufacturer standards. ${ }^{8,9}$

\section{Handheld Tonometer}

The handheld tonometer tip converts the applied force into an electrical signal, which is then processed by an algorithm to display an average IOP over several readings. The following technique was utilized to obtain measurements.

The device was calibrated prior to use. To calibrate, the activation button was pressed and held for 5 seconds until a beep sounded. After the 5 second button hold, the display showed [dn] on the screen. The device was then held vertical with the tip pointing to the floor for 15 seconds. Afterwards, the device beeped again and displayed [UP]. The device tip was pointed straight up into the air. If done properly, the device displayed [Pass]. The activate button was then pushed to put the device into applanation mode. If the calibration failed, these instructions were repeated.

When the investigator was ready to take an IOP measurement, an Ocu-Film + Tip Cover (Reichert, Depew, NY) was gently placed over the device tip. Of note, it was ensured that the tip cover was not too tight or the device will not measure properly. A drop of topical anesthetic was placed onto the eye. For corneal applanation tonometry, the patient was instructed to fixate straight ahead in primary gaze, and open both eyes. For scleral applanation, the patient was asked to look down and in, in order to adequately expose the superotemporal scleral for scleral tonometry measurement. When necessary, as required by a patient's specific anatomy, the upper lid was very gently raised to take great care not to push on the eye itself, as this could cause imprecise readings. In these cases, when it was necessary for the clinician to lift the upper lid, this was done for all measurements on that particular eye with identical technique to assure standardization. The tip of the device was gently touched to the surface of the eye. A chirp was heard as each measurement was taken. The device takes 10 measurements over the course of the gentle tapping on the eye. The device averages the measurements and displays a final IOP and confidence interval (CI). The higher the CI, the more accurate the measurement (ie 95\% CI designates a standard deviation of less than $5 \%$ of the number displayed as the IOP).

If the device was unable to get a measurement, the patient was asked to blink several times, and the measurement was attempted once again. After use, the tip cover was removed, and the device tip was cleaned with an alcohol wipe. ${ }^{8}$

\section{Pneumatonometer}

The pneumatonometer is a tonometer, which uses air flow to measure intraocular pressure. There is a constant flow of air through the device that is halted when the tip of the device is touched to the eye. The device will then push more air through until it flows again, breaking the seal created. At this point, a pressure sensor will record the amount of pressure, measured in $\mathrm{mmHg}$, required to match the IOP.

To calibrate, the Calibration Verifier was filled with water up to the $15 \mathrm{mmHg}$ line (Figure 1). The device was switched to "manual IOP mode". An eye was selected on the device screen (either OD or OS), and the tip of the device was touched to the Calibration Verifier membrane, making sure to align the tip of the probe such that the white part of the probe handle is between the red and black lines. At this point, the device provided an automated reading to verify proper calibration (should read 15 $\mathrm{mmHg}$ ).

The patient was instructed to slightly sit back, with chin up parallel to the floor (although the option exists to have a patient lie supine). A drop of topical anesthetic was instilled into the patient's eye. To take a measurement, the "manual IOP mode" option was selected on the device screen, and the eye being measured was selected. The device displayed a message when ready to measure. Holding the device tip like a pen, we instructed the patient to open both eyes wide and look in the appropriate desired position. When necessary, as required by a patient's specific anatomy, the upper lid was very gently raised to take great care not to push on the eye itself as this could cause imprecise readings. In these cases, when it was necessary 


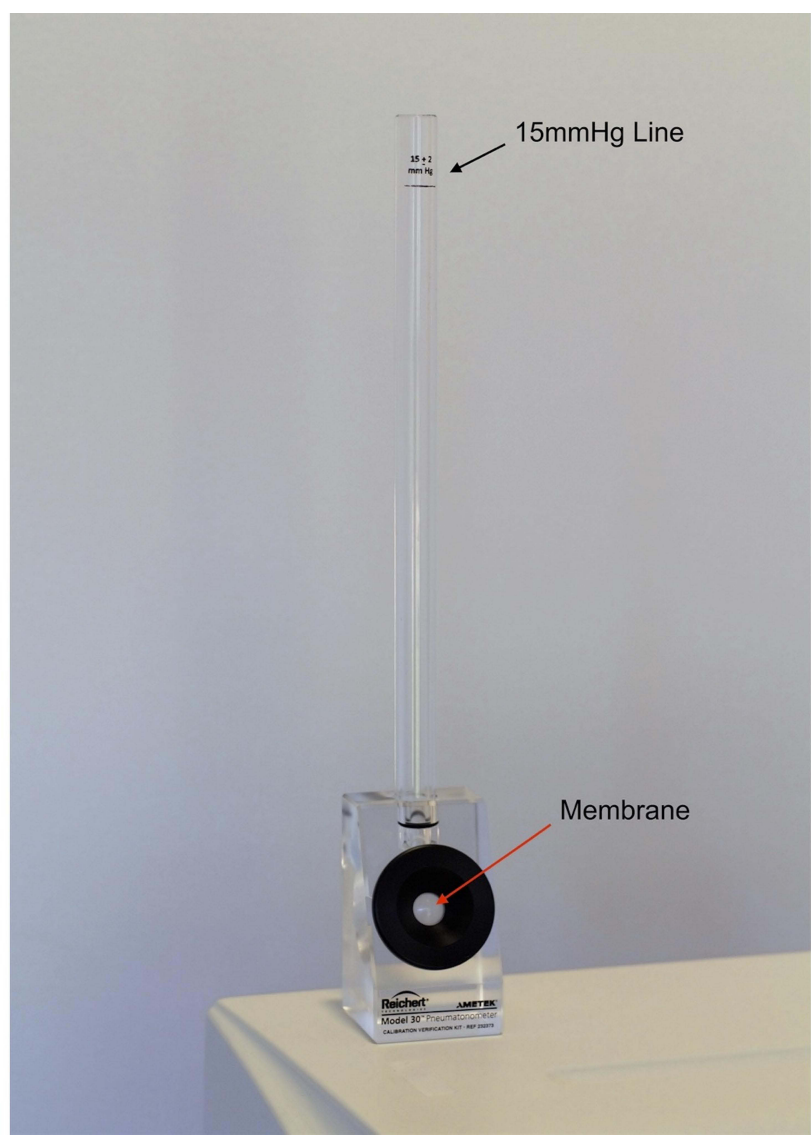

Figure I Calibration Verifier (Image courtesy of BostonSight, Needham MA).

for the clinician to lift the upper lid, this was done for all measurements on that particular eye with identical gentle technique to assure standardization. The sensor was gently moved towards the eye. As contact was made, the sensor handle was moved forward until the black line on the probe piston was hidden just under the white housing (Figure 2A and $\mathrm{B}$ ). The device will take measurements (at 40 per second) until the device tip is removed. The device emits a low-pitched tone when the standard deviation is less than $1.0 \mathrm{mmHg}$ for longer than 3 seconds, indicating accurate data. The device then displays an IOP and the accompanying standard deviation.

To clean, we removed the tip and membrane assembly from the probe after each patient. We separated the silicone membrane from the tip. To disinfect between uses, each piece was soaked in 3\% hydrogen peroxide for two separate 15 minute baths. The parts were then soaked in a bath of sterile normal saline for 15 minutes, rinsed thoroughly with sterile normal saline and allowed to completely air dry (25 or more minutes) prior to use. When ready for re-use, we reinstalled the tip and membrane on the device probe. The correct installation of the tip and membrane assembly is for the plastic tubing to be positioned halfway down the metal tube of the pneumatonometer (Figure 2B). ${ }^{9}$

\section{Statistical Analysis}

Statistical analysis was performed using Microsoft Excel and the Statistical Package for the Social Sciences (SPSS) program version 21.0 (IBM Corp. Armonk, New York, NY, USA). Normality was determined utilizing an online Shapiro-Wilk Test calculator (https://www. statskingdom.com/shapiro-wilk-test-calculator.html).

Continuous normally distributed variables are represented as mean \pm standard deviation. Continuous variables with non-normal distribution are reported with mean \pm standard deviation along with median (Interquartile range [IQR]). Any comparison of independent data sets, which includes variables with non-normal distribution, is analyzed for statistical significance with non-parametric testing utilizing a Mann Whitney $U$-test calculator (https://www.statskingdom.com/170median mann whitney.html). Comparison of paired data sets, which include variables with non-normal distribution, is analyzed for statistical significance with non-parametric testing utilizing a Wilcoxon Signed-Rank test calculator (https://www.statskingdom.com/175wilcoxon_signed_ ranks.html). Comparison of data sets with confirmed normality is analyzed via the applicable $T$-test (Paired $T$-test or Two Sample $T$-test [Welch's]). Significance was set to a P-value of $\leq 0.05$.

\section{Ethical Approval}

This study protocol was approved by the New England IRB (NEIRB), an Independent Institutional Review Board, on 7/14/2020, NEIRB File \# 1282180. All procedures and activities were performed in accordance with relevant state and local law and followed strict ethical obligations as set forth by the Declaration of Helsinki and Good Clinical Practice (GCP). All participants provided informed consent via an IRB approved informed consent document. The clinician completed institutionally required CITI Program training courses including "GCP for Clinical Trials with Investigational Drugs and Medical Devices (US FDA Focus)" and "Biomedical PI". All aspects of the study were conducted in compliance with the Health Insurance Portability and Accountability Act (HIPAA). 


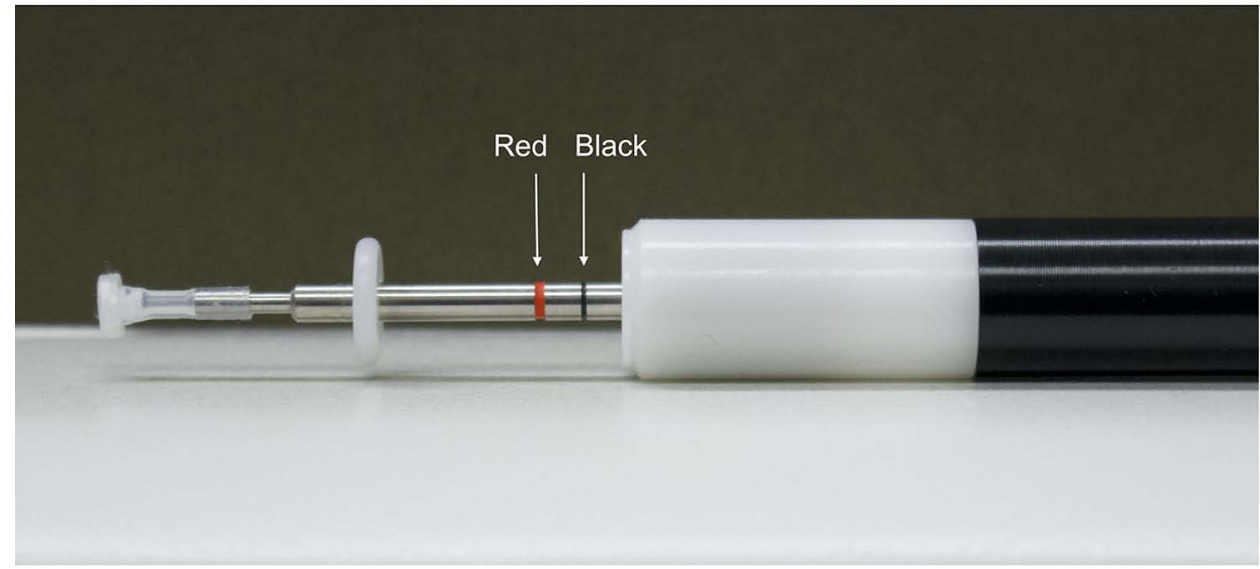

A

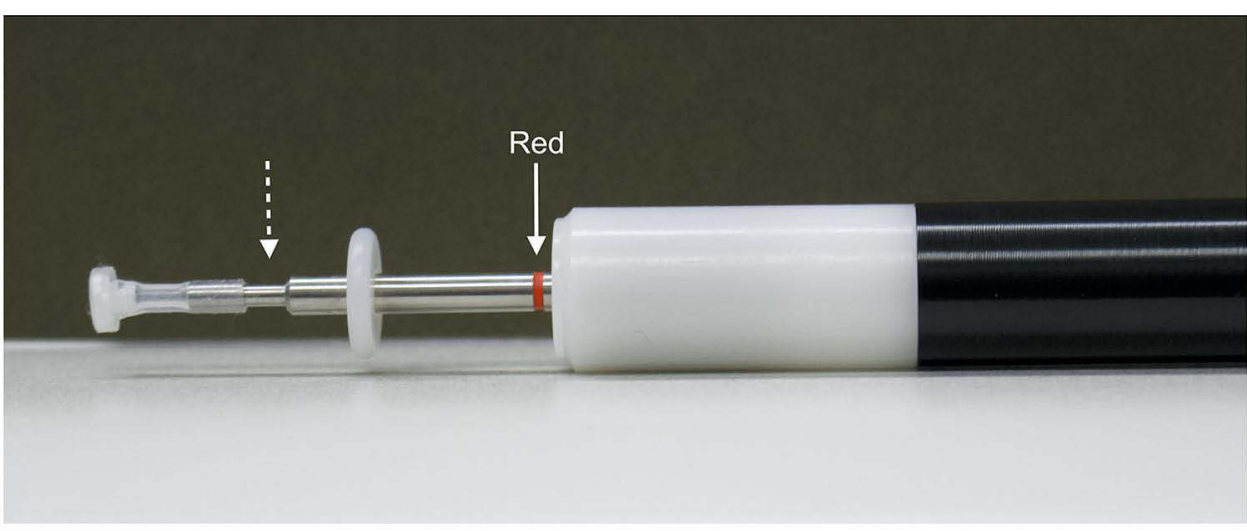

B

Figure 2 (A) Pneumatonometer probe: red and black lines on probe piston demarcate proper alignment for use. (Image courtesy of BostonSight, Needham MA). (B) Pneumatonometer probe shown in proper alignment position to achieve during use. The white housing of the probe handle must be oriented between the black and red lines for the measurement to be accurate. Additionally, note the dotted arrow showing proper placement of the re-useable plastic tip, halfway down the metal tube. (Image courtesy of BostonSight, Needham MA).

\section{Results}

\section{Demographics}

Data from forty eyes (20 patients) was collected in this study. Patients were consecutively assigned to either the handheld tonometer group (Subjects 1-10) or the pneumatometer group (Subjects 11-20). Age, gender, race and ethnicity were recorded for each individual in each cohort (Table 1). All subjects had been previously diagnosed with Keratoconus.

\section{Safety Parameters}

Corneal applanation tonometry with the handheld tonometer was used as a safety parameter in this study. All participants were required to have a corneal applanation IOP of $\leq 21 \mathrm{mmHg}$ to proceed with the study visit. The average IOP measured by corneal applanation with a handheld tonometer was $14.75 \pm 3.48 \mathrm{mmHg}$ in cohort one (handheld tonometer group) and $13.85 \pm 3.13 \mathrm{mmHg}$ in cohort two (pneumotonometer group). There was no significant difference in corneal applanation IOP between

Table I Subject Demographics

\begin{tabular}{|c|c|c|}
\hline & \multicolumn{2}{|c|}{ Cohort } \\
\hline & $\begin{array}{l}\text { Handheld } \\
\text { Tonometry }\end{array}$ & Pneumatonometry \\
\hline Age in years, mean \pm SD & $44.80 \pm 10.14$ & $50.80 \pm 7.74$ \\
\hline \multicolumn{3}{|l|}{ Gender, n (\%) } \\
\hline Male & $3(30)$ & $7(70)$ \\
\hline Female & $7(70)$ & $3(30)$ \\
\hline \multicolumn{3}{|l|}{ Race, ethnicity n (\%) } \\
\hline White, non-hispanic & $6(60)$ & $8(80)$ \\
\hline White, hispanic & $2(20)$ & $I(10)$ \\
\hline Black & $2(20)$ & $I(10)$ \\
\hline
\end{tabular}

Abbreviation: SD, standard deviation. 
the study groups $(p=0.395)$. No patients had any ocular surface changes at the conclusion of the study visit following multiple handheld tonometry or multiple pneumatonometry measurements.

\section{Measurements}

For every eye enrolled in the study, regardless of cohort, the investigator was able to achieve the goal of obtaining 3 reliable scleral applanated intraocular pressure measurements. This was achieved by a mean $3.45 \pm 0.69$, median (IQR) of 3 (3 to 4) attempts with handheld tonometry and a mean $3.2 \pm 0.41$, median (IQR) of 3 (3 to 3 ) attempts with pneumatonometry. There was no significant difference in the number of attempts needed to achieve 3 reliable measurements when comparing the handheld tonometer and pneumotonometer groups $(p=0.245)$. "Reliable" was set at $90 \%$ or greater confidence interval (CI) for each IOP measurement for the handheld tonometry group. There was an average confidence interval of $94.375 \pm 1.11 \%$ with a median (IQR) of 95 (94.375 to $95) \%$ across all reliable measurements in the handheld tonometry group. "Reliable" was set at a standard deviation of $2 \mathrm{mmHg}$ or less for each IOP measurement of the pneumatonometry group. There was an average standard deviation of $0.468 \pm 0.19 \mathrm{mmHg}$ across all reliable measurements in the pneumatonometry group.

For an individual eye, the three reliable intraocular pressure measurements were averaged and reported as the mean scleral IOP for that eye. These mean scleral IOPs were then used to calculate the average scleral IOP across the cohort, which was $24.23 \pm 8.59 \mathrm{mmHg}$ with a median (IQR) of 21 (18.67 to 25.42$) \mathrm{mmHg}$ in the handheld tonometer group and $22.32 \pm 6.41 \mathrm{mmHg}$ in the pneumatonometer group, not a statistically significant difference between the groups $(p=0.86)$. The mean change from the first to the third reliable intraocular pressure measurement in the handheld tonometer group was $-0.8 \pm 5.85 \mathrm{mmHg}(\mathrm{p}=0.55)$. The mean change from the first to the third reliable intraocular pressure measurement in the pneumatonometer group was -1.26 $\pm 3.38 \mathrm{mmHg}$ with a median (IQR) of 2.25 ( -2.25 to 4 ) $\mathrm{mmHg}(\mathrm{p}=0.12)$. There was no statistically significant change in either group from the first to the third reliable intraocular pressure measurement.

For an individual eye, a standard deviation was calculated for the three reliable intraocular pressure measurements obtained. Using these standard deviation values for each eye, a mean standard deviation was calculated for each cohort and was found to be $\pm 2.92 \mathrm{mmHg}$ with a median (IQR) of 2.62 (1.68 to 3.53 ) $\mathrm{mmHg}$ in the handheld tonometer group and $\pm 1.98 \mathrm{mmHg}$ in the pneumatonometer group, not a statistically significant difference between the groups $(p=0.07)$.

For an individual eye, a range was calculated for the three reliable intraocular pressure measurements obtained. Using these values for each eye, a mean IOP range was calculated for each cohort and was found to be $5.5 \pm 3.80$ mmHg with a median (IQR) of 5 (3 to 7) $\mathrm{mmHg}$ for the handheld tonometer group and $3.71 \pm 1.12 \mathrm{mmHg}$ in the pneumatonometer group, not a statistically significant difference between the groups $(p=0.06)$.

\section{Discussion}

Over recent years, a question has arisen in the scleral lens community whether application and daily wear of a scleral lens or PD can affect intraocular pressure in any manner. Several clinical studies have attempted to investigate this issue to determine if any adjustment to clinical recommendations, indications or contraindications for scleral lens wear should occur. At the very heart of trying to address this complex issue is a significant obstacle for data collection; there is currently no agreement upon standardized instrument with known precision (reproducibility) for measuring intraocular pressure on the sclera, while a PD or scleral lens is applied.

No one to date has particularly investigated the pathophysiology of how application of a scleral lens to the eye could increase (or decrease) intraocular pressure. Perhaps a smaller, tighter scleral lens could mechanically result in circulatory restriction and therefore increase episcleral venous pressure, although no evidence yet exists to support this. Additionally, long-term scleral lens fitters who have fit these lenses over decades have not reported anecdotally, in literature, symposia, poster presentations or otherwise an increased incidence of glaucoma in their patient population.

Overall, studies thus far investigating a possible relationship between scleral lens wear and IOP have been equivocal. ${ }^{10-12}$ Central to investigating any potential relationship, is a very basic question of what reliability are traditional tonometry options when applanated on the sclera, rather than the cornea, while a subject is wearing a PD or scleral lens. Of note, it is already widely agreed upon that scleral tonometry is significantly inaccurate when compared to corneal applanation tonometry. ${ }^{13,14}$ Some studies have postulated a correction factor to convert 
scleral tonometry to comparative corneal applanation tonometry values, but reliability and agreed upon convention does not exist. ${ }^{15}$ Previous studies have evaluated peripheral IOP measurements with various instruments but have concentrated on investigating accuracy rather than precision. For instance, Kolin et al investigated a comparison of scleral versus corneal handheld tonometry. Only one corneal and one scleral measurement was taken in each eye. Investigators concluded scleral tonometry was inaccurate when compared to corneal results. However, no comment was made about scleral tonometry precision, nor could this be evaluated having only taken one measurement on the sclera. ${ }^{13}$ Badakere et al investigated the agreement between corneal Goldmann applanation and corneal, limbal, and scleral handheld tonometer measurements. ${ }^{16}$ While investigators discussed a wide $95 \%$ limit of agreement when analyzing limbal and scleral measurements, this study did not report confidence intervals of tonometer readings and did not include pneumotonometry analysis. Our study and the data presented here add to the current literature by evaluating the precision of handheld tonometry and pneumatonometry, while a scleral lens is applied to the eye, a model that is currently frequently being used in prospective clinical trials to evaluate for any association between scleral lens wear and eye pressure.

The data presented in this report suggest that neither handheld tonometry nor pneumatonometry provide adequate precision/reproducibility to be an instrument of choice when collecting scleral IOP data for a subject while wearing a PD or scleral lens. Although 3 measurements that the device considered "reliable" were readily obtained in an average of 3.45 and 3.2 attempts in the handheld tonometer and pneumatonometry cohorts, respectively, the precision of these "reliable" measurements on an individual subject was poor. Of note, 10 investigator attempts were the maximum allowable in the pneumatonometer cohort, as opposed to a maximum of 5 investigator attempts in the handheld tonometer cohort. This difference was in place due to the recognition that in general, clinicians have limited experience with a pneumatonometer compared to a handheld tonometer. In the end, a single individual clinician who was well versed and experienced in the use of both the handheld tonometer and pneumatonometer conducted all study visits.

Previous studies have taken varied approaches to analyzing IOP in scleral lens wearers. A majority of studies take two to three consecutive IOP measurements for each eye at each specific time point and average the results, but of note, such studies typically make no comment regarding the confidence interval or standard deviation readout from the instrument itself (the instrument's assessment of the data reliability). ${ }^{1,2,4,6,12}$ We believe reporting on this data set is essential as this is how a clinician in a typical office setting would determine whether a measurement taken individually was "reliable" by manufacturer's standards. Each individual pressure measurement deemed "reliable" and analyzed in each cohort for each eye in the handheld tonometry group had a CI of $90 \%$ or greater (mean $94.38 \%$ ) and in the pneumatonometry group had a standard deviation less than $2 \mathrm{mmHg}$ (mean 0.468). Similar average IOP measurements were achieved in each cohort; however, the average standard deviation for the measurements was \pm 2.92 in the handheld tonometer group and \pm 1.98 in the pneumatonometer group. This sizeable deviation along with the wide average IOP range measured for each individual eye (average range $5.5 \pm$ $3.80 \mathrm{mmHg}$ for the handheld tonometer group and 3.71 \pm 1.12 in the pneumatonometer group) underscore the lack of reproducibility these instruments have when used in this manner. For comparison, Dielemans et $\mathrm{al}^{17}$ reported a mean standard deviation of only $0.8 \mathrm{mmHg}$ for three consecutive IOP measurements via Goldmann corneal applanation tonometry, the agreed upon gold standard of tonometry.

There are certainly limitations to note in the presented data set. The number of patients in this pilot study was intentionally limited to serve as a first evaluation of the general precision quality of these techniques and applications of tonometry. We believe the data is significant enough to help guide decisions about utilizing these instruments for future scleral lens IOP studies. However, additional larger trials could certainly be beneficial, particularly to mitigate any effect data outliers could have on statistical analysis of a smaller study population size.

A second limitation relates to the population pathology. All patients in this study had keratoconus, which may affect the results. It is possible that scleral tissue is less rigid in these subjects and results in more variability in tonometry measurement to measurement. Additional studies on normal eyes or eyes without pathologic tissue (ie dry eye rather than keratoconus) may prompt different results. Of note, reproducibility of traditional corneal applanation tonometry readings (ie with a handheld tonometer) in keratoconus patients is not generally considered 
problematic in standard clinical care, although accuracy may be suspect specifically at later stages given corneal irregularities and variable corneal thickness. ${ }^{18}$

One additional concern regarding repetitive measurements on the same eye in the same location is artificially altering the IOP. A concern could reasonably be raised that with each repetitive measurement with pneumatonometry or handheld tonometry we may be unintentionally performing ocular massage which may have the unintended effect of lowering IOP. ${ }^{19}$ This may indeed be a valid consideration in this data set. While not statistically significant, the average IOP did decrease for both the handheld tonometer group (average reduction $0.8 \pm 5.85$ $\mathrm{mmHg}, \mathrm{p}=0.548$ ) and the pneumatonometry group (average reduction $1.26 \pm 3.38 \mathrm{mmHg}, \mathrm{p}=0.112$ ) when comparing the first and the third reliable intraocular pressure measurements. The risk of this possibly unintended effect should be taken into account in future study protocol designs.

One of the advantages of using the handheld tonometer or pneumatonometer is the safety of these instruments. It is quite rare to have adverse events such as corneal abrasion or irritation from utilization of these instruments and that was born out in our data set, with no patients having any change in exam pre- and post-multiple tonometry measurements.

Tremendous care and diligence was taken in standardizing and reproducing the set-up and techniques with each tonometry instrument, carefully referencing the instrument manuals for both devices. Paramount for the determination of device precision is a standardized methodology for utilizing the tonometry instruments according to manufacturer's recommendations. Comparing data between studies or between subjects is invalid without such standardization. Proper use and calibration of the handheld tonometer and pneumatonometer is detailed in the methods section, not only as a reference for this study but also as a reference for future studies to use a standardized approach for IOP data collection.

Common pitfalls when using a handheld tonometer include failure to calibrate the device prior to use, too tight application of the disposable tip cover and failure to recognize known loss of accuracy in the extreme ranges of IOP measurements. ${ }^{20}$ Common pitfalls when using a pneumatonometer include failure to calibrate the device prior to use, improper placement of the tip and membrane assembly tubing too far down on the metal tube (proper placement is for the plastic tubing to be positioned halfway down the metal shaft), exerting too intense pressure with the tip upon application on the eye, and damage to the tip membrane when handling (such as during disinfection). When manipulation of the lids is necessary, unintentional pressure on the globe is not unusual, and the clinician must be quite gentle in order to prevent artificially elevating the pressure readings when utilizing any tonometer. Variable unintentional pressure on the globe while lifting the lids could contribute to loss of precision within a group of measurements on a single eye and risk becoming a confounding variable. Therefore, if lifting the upper lid was necessary for a particular eye in this study, the lid was lifted for all measurements on that particular eye, always with great gentle care taken to not exert any pressure on the globe. Future studies could track this data point (whether the lids were manually lifted) to discern any precision difference in such groups.

Most clinicians have minimal experience with the use of a pneumatonometer, and therefore in this report we highlight both the proper usage and common errors for all clinicians considering utilizing this instrument, particularly those considering the device for scleral lens IOP research applications. For both devices, standardization of the location of applanation on the eye was considered of great importance. Applanating over a muscle insertion, for instance, could potentially be a confounding variable for reproducibility. Therefore, the superotemporal location approximately $1 \mathrm{~mm}$ posterior to the PD edges was chosen. Varying location of applanation when comparing study to study is an additional important confounding variable making it difficult to accumulate comparable data. This is particularly worrisome if future metaanalyses are performed.

Future studies should consider investigating the precision of other standard tonometry instruments for this application. As well, other avenues could be undertaken to continue to investigate any association between scleral lens wear and IOP. For instance, use of implantable intraocular pressure monitors in a prospective study design would be an appropriate application of such breakthrough technology, most of which is still in preclinical or early clinical trials. Retrospectively reviewing a large cohort of longitudinal data on scleral lens wearers could be a rewarding approach to explore the lifetime risk of glaucomatous pathology relative to the general population. Prospective analysis of traditional long-term glaucoma endpoints such as optic nerve cupping, optic nerve optical coherence tomography, automated visual 
fields or newer glaucoma markers such as optical coherence tomography-angiography ${ }^{21}$ may be alternative pathways to investigating if there is any association between glaucoma risk and scleral lens wear. These endpoints would allow investigators to move away from the surrogate endpoint of IOP, which presents notable obstacles when one wishes to measure IOP during scleral lens wear.

\section{Conclusion}

The data obtained and analyzed in this report raises concerns about the precision of using handheld tonometry or pneumatonometry to investigate the association between intraocular pressure and scleral lens wear. Ongoing research into alternative instrumentation and/or novel research models is needed. Ultimately, the future use of implantable intraocular pressure monitors may be the most promising method for investigation, providing an avenue that would eliminate the precision concerns, which are present when utilizing standard tonometry instruments developed for corneal applanation in a non-standard manner on the sclera.

\section{Data Sharing Statement}

The data that support the findings of this study are available from the author, $\mathrm{AG}$, upon reasonable request. The authors confirm that the data supporting the findings of this study are available within the article.

\section{Acknowledgments}

The authors acknowledge the contributions of Kellen Riccobono, OD, FAAO (Department of Optometry, New England College of Optometry) to the creation of the study protocol and Stephanie Derby, ophthalmic technician (BostonSight), for photographing the color images (Figures 1 and 2A, B).

\section{Author Contributions}

All authors made a significant contribution to the work reported, whether that is in the conception, study design, execution, acquisition of data, analysis and interpretation, or in all these areas; took part in drafting, revising or critically reviewing the article; gave final approval of the version to be published; have agreed on the journal to which the article has been submitted; and agree to be accountable for all aspects of the work.

\section{Funding}

This research received no specific grant from any funding agency in the public, commercial, or not-for-profit sectors. It was supported by BostonSight institutional resources.

\section{Disclosure}

D. Brocks is a salaried employee of BostonSight, Needham, MA, where BostonSight PROSE treatment was developed, and where PROSE devices and BostonSight SCLERAL lenses are manufactured. None of the authors have a proprietary interest in PROSE treatment, the prosthetic devices used in PROSE treatment, or BostonSight SCLERAL lenses. The authors report no other conflicts of interest in this work.

\section{References}

1. Fogt JS, Nau CB, Schornack M, Shorter E, Nau A, Harthan JS. Comparison of pneumatonometry and transpalpebral tonometry measurements of intraocular pressure during scleral lens wear. Optom Vis Sci. 2020;97(9):711-719. doi:10.1097/OPX.0000000000001574

2. Nau CB, Schornack MM, McLaren JW, Sit AJ. Intraocular pressure after 2 hours of small-diameter scleral lens wear. Eye Cont Lens. 2016;42(6):350-353. doi:10.1097/ICL.0000000000000214

3. Kramer EG, Vincent SJ. Intraocular pressure changes in neophyte scleral lens wearers: a prospective study. Cont Lens Anterior Eye. 2020;43(6):609-612. doi:10.1016/j.clae.2020.05.010

4. Formisano M, Franzone F, Alisi L, Pistella S, Spadea L. Effects of scleral contact lenses for keratoconus management on visual quality and intraocular pressure. Ther Clin Risk Manag. 2021;17. doi:10.2147/TCRM.S293425

5. Fedotova K, Zhu W, Astakhov S, Novikov SA, Grabovetskiy VR, Nikolaenko VP. Intraocular pressure with miniscleral contact lenses. Vestn Oftalmol. 2021;137(2):52-58. doi:10.17116/oftalma20211 3702152

6. Michaud L, Samaha D, Giasson CJ. Intra-ocular pressure variation associated with the wear of scleral lenses of different diameters. Cont Lens Anterior Eye. 2019;42(1):104-110. doi:10.1016/j.clae.2018. 07.004

7. Agranat JS, Kitos NR, Jacobs DS. Prosthetic replacement of the ocular surface ecosystem: impact at 5 years. $\mathrm{Br} J$ Ophthalmol. 2016;100(9):1171-1175. doi:10.1136/bjophthalmol-2015-307483

8. Tono-Pen AVIA ${ }^{\circledR}$ Tonometer User's Guide [Manual on the Internet]. AMETEK, Inc; 2017. Available from: http://doclibrary. com/MSC167/PRM/68E3892-Rev-J-UG-AVIA4540.pdf. Accessed September 14, 2021.

9. Model 30 TM Pneumatonometer User's Guide [Manual on the Internet]. AMETEK, Inc; 2017. Available from: https://lombartinstru ment.com/store/pub/media/wysiwyg/Model-30-Users-Guide.pdf. Accessed September 14, 2021.

10. Aitsebaomo AP, Wong-Powell J, Miller W, Amir F. Influence of scleral lens on intraocular pressure. J Cont Lens Res Sci. 2019;3(1): e1-e9. doi:10.22374/jclrs.v3i1.34

11. Walker MK, Pardon LP, Redfern R, Patel N. IOP and optic nerve head morphology during scleral lens wear. Optom Vis Sci. 2020;97 (9):661-668. doi:10.1097/OPX.0000000000001567

12. Shahnazi KC, Isozaki VL, Chiu GB. Effect of scleral lens wear on central corneal thickness and intraocular pressure in patients with ocular surface disease. Eye Cont Lens. 2020;46(6):341-347. doi:10.1097/ICL.0000000000000670 
13. Kolin T, Wedemeyer LL, Kolin E, Braun Y. Comparison of scleral and corneal Tono-Pen readings. $J$ Am Assoc Pediatr Ophthalmol Strabismus. 2003;7(4):291-292. doi:10.1016/S1091-8531(03)00147-2

14. Khan JA. Comparison of oculab Tono-Pen readings obtained from various corneal and scleral locations. Arch Ophthalmol. 1991;109 (10):1444. doi:10.1001/archopht.1991.01080100124057

15. Kuo DS, Ou Y, Jeng BH, et al. Correlation of serial scleral and corneal pneumatonometry. Ophthalmology. 2015;122(9):1771-1776. doi:10.1016/j.ophtha.2015.05.033

16. Badakere SV, Choudhari NS, Rao HL, Chary CR, Garudadri CS, Senthil S. Comparison of scleral Tono-Pen intraocular pressure measurements with Goldmann applanation tonometry. Optom Vis Sci. 2018;95(2):129-135. doi:10.1097/OPX.0000000000001174

17. Dielemans I, Vingerling JR, Hofman A, Grobbee DE, de Jong PTVM. Reliability of intraocular pressure measurement with the Goldmann applanation tonometer in epidemiological studies. Graefes Arch Clin Exp Ophthalmol. 1994;232(3):141-144. doi:10.1007/BF00176782
18. Altinkaynak H, Kocasarac C, Dundar H, et al. Which tonometry in eyes with keratoconus? Eye. 2016;30(3):431-437. doi:10.1038/eye.2015.248

19. Lam AKC, Chen D. Effect of ocular massage on intraocular pressure and corneal biomechanics. Eye. 2007;21(9):1245-1246. doi:10.1038/ sj.eye. 6702928

20. Ruland K, Olayanju J, Borras T, Grewal DS, Fleischman D. Accuracy of Tonopen versus iCare in human cadaveric eyes with edematous corneas over a wide range of intraocular pressures. $J$ Glaucoma. 2019;28(5):e82-e85. doi:10.1097/IJG.0000000000001162

21. Nguyen K, Lee J, Richter GM, Chiu GB. The effect of scleral lens wear on optic disc perfusion measured by optical coherence tomography angiography (OCTA). Global Specialty Lens Symposium. USC Roski Eye Institute; 2021.

\section{Clinical Optometry}

\section{Publish your work in this journal}

Clinical Optometry is an international, peer-reviewed, open access journal publishing original research, basic science, clinical and epidemiological studies, reviews and evaluations on clinical optometry. All aspects of patient care are addressed within the journal as well as the practice of optometry including economic and business analyses. Basic and clinical research papers are published that cover

\section{Dovepress}

all aspects of optics, refraction and its application to the theory and practice of optometry. The manuscript management system is completely online and includes a very quick and fair peer-review system, which is all easy to use. Visit http://www.dovepress.com/ testimonials.php to read real quotes from published authors. 